\title{
Técnica para correção de truncus arteriosus tipos I e II sem condutos extracardíacos
}

\author{
Miguel BARBERO-MARCIAL*, Arlindo A. RISO*, Geraldo VERGINELLI*, Fúlvio PILEGGı*, Adib D. JATENE*
}

\section{RBCCV 44205-72}

BARBERO-MARCIAL, M.; RISO, A. A.; VERGINELLI, G.; PILEGGI, F.; JATENE, A. D. - Técnica para correçăo de truncus arteriosus tipos I e II sem condutos extracardiacos. Rev. Bras. Cir. Cardiovasc., 4(1): 1-8; 1989.

RESUMO: Uma nova técnica para correçăo cirúrgica do truncus arteriosus (TA) sem o uso de conduto extracardiaco foi realizada em 7 pacientes, senao 6 do tipo I, e do tipo II em 1 paciente; a idade variou de 2 a 9 meses. O tronco comum foi septado com retalho, dividindo-o em uma posiçăo aórtica e outra pulmonar; a comunicação interventricular (CIV) foi fechada através de ventriculotomia; anastomose direta entre as artérias pulmonares e o ventrículo direito foi realizada, sendo a parede anterior construída com retalho de pericárdio bovino com válvula monocúspide. Houve apenas 1 óbito no pós-operatório. Nos 6 pacientes restantes, a relaçāo entre as pressōes sistólicas ventrículo direito/ventrículo esquerdo (VD/VE) foi menor que 0,51 em 5 pacientes; em 1 paciente, essa relação foi de 0,60 , associada a CIV residual. Todos os pacientes encontram-se em classe funcional I (NYHA), em um período de 1 a 14 meses de pós-operatório. Baseados nestes resultados, propomos esta técnica para pacientes com TA tipo I ou II, no primeiro ano de vida.

DESCRITORES: truncus arteriosus, cirurgia.

\section{INTRODUÇĀO}

Pacientes portadores de truncus arteriosus apresentam alta mortalidade nos primeiros dias de vida ${ }^{9}$. Devido a este fato, há um consenso geral de que o tratamento cirúrgico seja precoce. A cirurgia paliativa para a restrição do fluxo pulmonar apresenta alta morbidade e mortalidade ${ }^{8}$. A correçăo precoce com conduto valvulado extracardíaco, apesar de ter sido um grande progresso, apresenta, como desvantagens, o alto risco operatório e a necessidade de troca do conduto, seja pelo crescimento da criança, ou pela obstrução'.

Neste trabalho, apresentamos uma nova técnica de correçăo sem o uso de conduto extracardíaco.

\section{CASUISTICA E MÉTODOS}

Sete pacientes consecutivos portadores de truncus arteriosus tipos I e II foram submetidos a correçăo cirúrgica, no Instituto do Coraçăo, entre agosto de 1987 e julho de 1988 (Tabela 1). O peso variou de 3,2 a 6,4 kg (média 4,7) e a idade na operação, de 2 a 9 meses. Todos os pacientes apresentavam sinais e sintomas de insuficiência cardíaca severa e cianose. O estudo ecocardiográfico pré-operatório diagnosticou truncus arteriosus tipo I em 6 pacientes e tipo II em 1 paciente. Somente este último paciente foi submetido a cateterismo pré-operatório e o diagnóstico de truncus arteriosus tipo II foi confirmado.

Trabalho realizado no Instituto do Coraçăo do Hospital das Clínicas da Faculdade de Medicina da Universidade de São Paulo. Săo Paulo, SP, Brasil.

Apresentado ao 16: Congresso Nacional de Cirurgia Cardlaca. Săo Paulo, SP, 7 e 8 de abril, 1989

Laureado com o "Prêmio Nacional de Cirurgia Cardíaca - 1989".

* Do Instituto do Coraçăo do Hospital das Clínicas da Faculdade de Medicina da Universidade de Săo Paulo.

Endereço para separatas: Miguel Barbero-Marcial. Av. Dr. Enéas de Carvalho Aguiar, 44. Divisão Cirúrgica. 05403 São Paulo, SP, Brasil. 
TABELA 1

DADOS CLINIICOS

\begin{tabular}{|c|c|c|c|c|c|c|}
\hline Pac. & $\begin{array}{l}\text { Idade } \\
\text { (meses) }\end{array}$ & $\begin{array}{l}\text { Peso } \\
(\mathrm{kg})\end{array}$ & $\begin{array}{l}\text { Tipo de } \\
\text { truncus }\end{array}$ & $\begin{array}{l}\text { Insuficiência da valva } \\
\text { truncal pré-operatória } \\
\text { (ECO) }\end{array}$ & Técnica operatoria & $\begin{array}{c}\text { Evolução } \\
\text { Tempo e classe funcional } \\
\text { (NYHA) }\end{array}$ \\
\hline 01 & 2 & 3,9 & 1 & Moderada & $\begin{array}{l}\text { Anastomose direta VD-TP } \\
\text { fechamento de CIV }\end{array}$ & 14: mês P.O. bem, classe I \\
\hline 02 & 8 & 6,4 & 1 & Pequena & $\begin{array}{l}\text { Anastomose direta VD-TP } \\
\text { fechamento de CIV }\end{array}$ & 11: mês P.O. bem, classe I \\
\hline 03 & 9 & 3,9 & 1 & Ausente & $\begin{array}{l}\text { Anastomose direta VD-TP } \\
\text { Ampliação da CIV túnel } \\
\text { VE - tronco comum }\end{array}$ & $7^{\circ}$ mês P.O. bem, classe I \\
\hline 04 & 8 & 4,5 & 1 & Pequena & $\begin{array}{l}\text { Anastomose direta VD-TP } \\
\text { Fechamento de CIV }\end{array}$ & $4:$ mês P.O. bem, classe I \\
\hline 05 & 6 & 3,5 & II & $\begin{array}{l}\text { Moderada, CIV } \\
\text { restritiva tronco } \\
\text { comum no VD }\end{array}$ & $\begin{array}{l}\text { Ampliaçăo da CIV túnel } \\
\text { VE - tronco comum. } \\
\text { Interposiçăo da aurícula e APE }\end{array}$ & Morte súbita no P.O. \\
\hline 06 & 4 & 6,0 & 1 & Pequena & $\begin{array}{l}\text { Anastomose direta VD-TP } \\
\text { Fechamento de CIA }\end{array}$ & $2^{\circ}$ mês P.O. bem, classe I \\
\hline 07 & 3 & 3,2 & 1 & Ausente & $\begin{array}{l}\text { Anastomose direta VD-TP } \\
\text { Fechamento de CIA }\end{array}$ & 1: mês P.O. bem, classe I \\
\hline
\end{tabular}

VD: ventrículo direito; TP: tronco pulmonar; CIV: comunicaçăo interventricular; VE: ventrículo esquerdo; APE: artéria pulmonar esqueda; P.O.: pós-operatório.

A operação é realizada através de toracotomia mediana; as artérias pulmonares são dissecadas amplamente e mobilizadas; as veias cavas, canuladas e a cânula aórtica, colocada próxima à emergência do tronco braquiocefálico.

É estabelecida a circulação extracorpórea, injetada solução cardioplégica e as câmaras esquerdas, drenadas através do forame oval ou do septo atrial.

Nos 6 pacientes com truncus tipo I, foi realizada uma incisão longitudinal na artéria pulmonar esquerda próximo à sua face ântero-superior (Figura 1a). Essa incisão foi estendida para baixo, verticalmente, na direçăo do seio de Valsalva esquerdo do tronco comum. Após a identificação do orifício da artéria pulmonar direita e do óstio coronariano esquerdo, um retalho de pericárdio bovino foi suturado, dividindo o truncus arteriosus em duas partes: aorta e artérias pulmonares. Para isto, a sutura foi iniciada próxima ao anel da valva truncal, deixando-se o seio da Valsalva esquerdo no componente pulmonar (Figura 1b). A sutura posterior do retalho foj feita entre o óstio coronariano esquerdo e o orifício de origem da artéria pulmonar direita. É preciso ressaltar que o retalho não deve ser muito grande, pois a pressão aórtica pode abaulá-lo sobre 0 orifício da artéria direita, obstruindo-o.

Uma ventriculotomia direita é realizada, iniciando-se logo abaixo do seio da Valsalva esquerdo, seguindo um trajeto oblíquo para baixo e para a esquerda. As trabé- culas cárneas geralmente presentes nas bordas da incisão são seccionadas. A comunicação interventricular é fechada com retalho de pericárdio bovino. Em 1 paciente (caso n: 3), a comunicação interventricular era restritiva, necessitando ampliação de $1 \mathrm{~cm}$ na borda ântero-superior. Após isto, a extremidade inferior da incisão da artéria pulmonar esquerda é tracionada para baixo e suturada à borda oblíqua esquerda da ventriculotomia, através de pontos separados em $U$, resultando uma linha de sutura praticamente horizontal (Figura 1c).

Como próxima etapa, um retalho de pericárdio bovino com válvula monocúspide é suturado nas bordas arterial e ventricular, com o propósito de construir a parede anterior da nova via de saída do ventrículo direito (Figuras 1d e 1e).

No paciente portador de truncus arteriosus tipo II (caso $\mathrm{n}: 5$ ), algumas variaçōes técnicas foram feitas (Figura 2). As artérias pulmonares, que se originavam da face posterior do tronco comum, foram separadas desse tronco, e o orifício foi fechado com retalho de pericárdio bovino. A comunicação inteventricular, restritiva em relaçăo ao anel truncal, foi ampliada na sua porçăo ânterosuperior. Devido à grande dextroposiçāo do tronco, a nova via de saída ventrículo esquerdo-aorta foi construída com 2 retalhos de pericárdio bovino, um superior e outro inferior, de tal maneira que a linha de sutura resultante abaulasse para a cavidade do ventrículo direito. 
BARBERO-MARCIAL, M.; RISO, A. A.; VERGINELLI, G.; PILEGGI, F.; JATENE, A. D. - Técnica para correçăo de truncus arteriosus tipos I e II sem condutos extracardiacos. Rev. Bras. Cir. Cardiovasc., 4(1): 1-8, 1989.

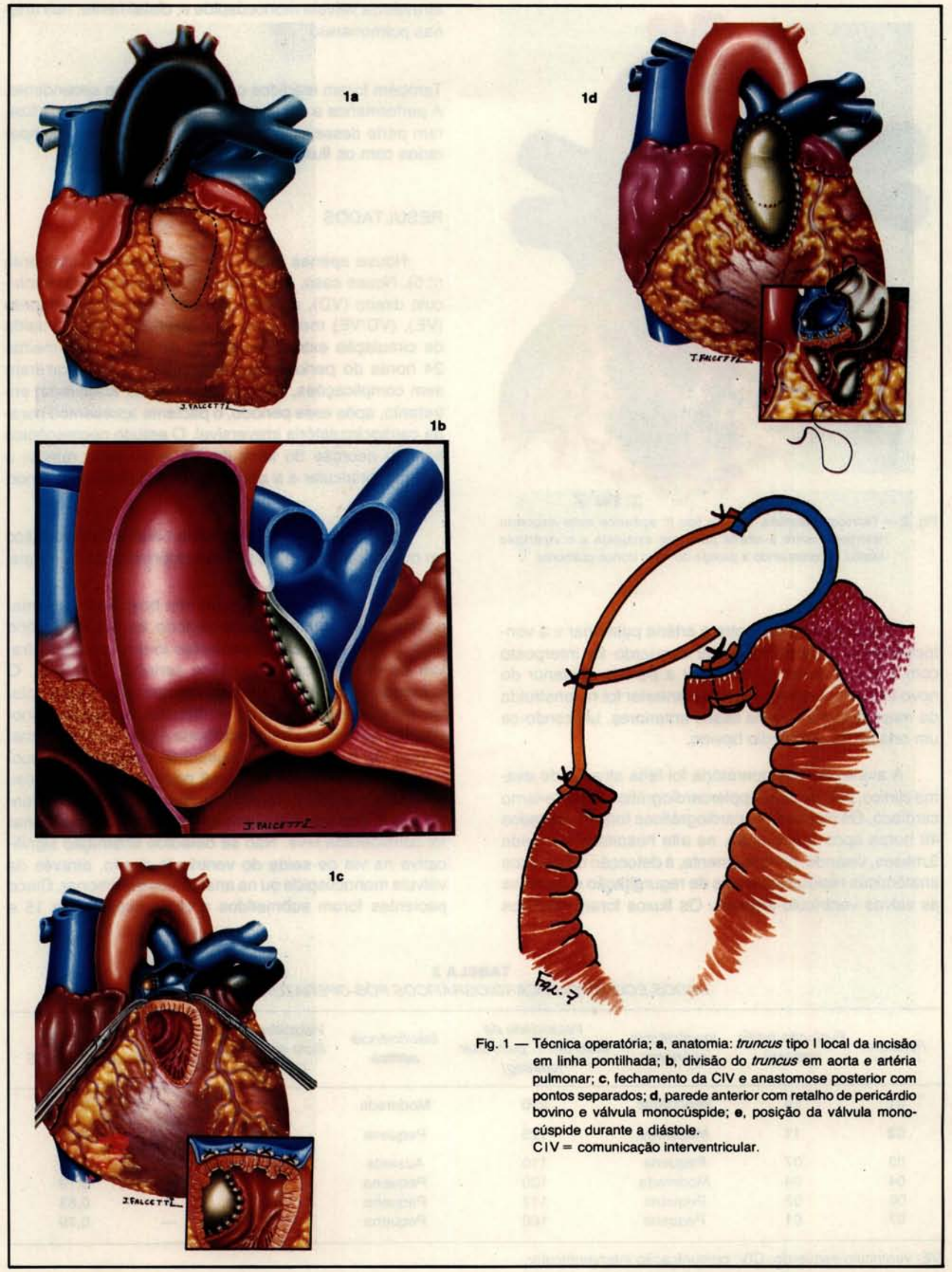




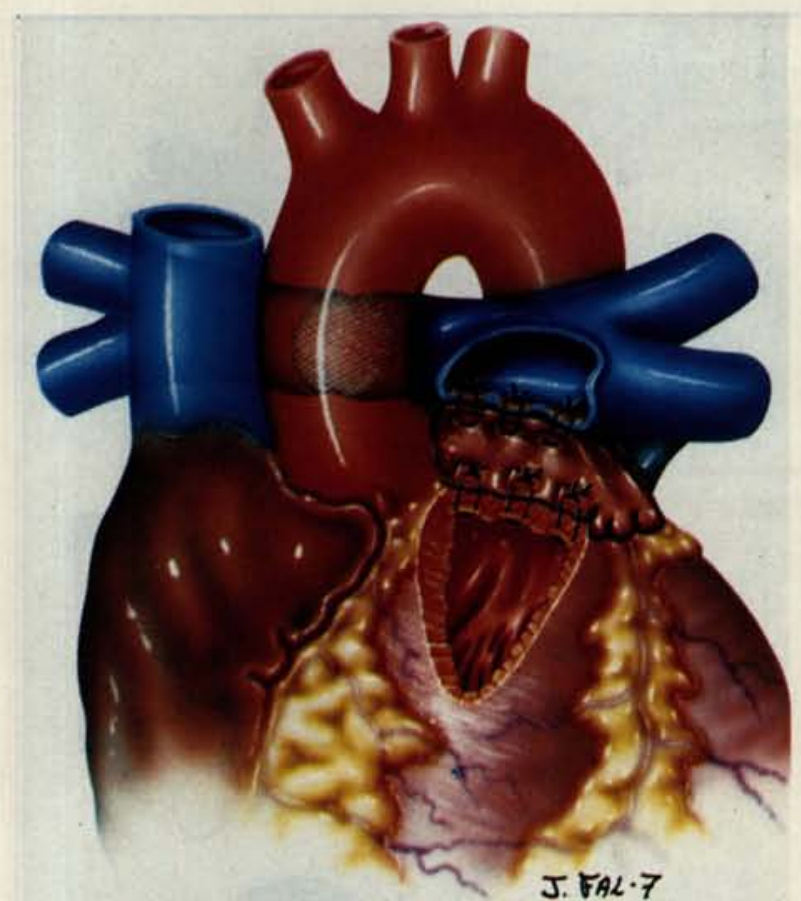

Fig. 2-Técnica operatória, truncus tipo II: apêndice atrial esquerdo interposto entre a artéria pulmonar esquerda e o ventriculo direito, reconstruindo a parede do novo tronco pulmonar.

Devido à distância entre a artéria pulmonar e a ventriculotomia, o apêndice atrial esquerdo foi interposto com o propósito de se construir a parede posterior do novo tronco pulmonar. A parede anterior foi reconstruída da mesma forma que os casos anteriores, utilizando-se um retalho de pericárdio bovino.

A avaliação pós-operatória foi feita através de exame clínico, estudo зcodopplercardiográfico e cateterismo cardíaco. Os estudos ecocardiográficos foram realizados 48 horas após a operação, na alta hospitalar e a cada 3 meses, visando, principalmente, à detecção de defeitos anatômicos residuais e sinais de regurgitação em ambas as valvas ventrículo-arteriais. Os fluxos foram medidos através da válvula monocúspide e, distalmente, nas artérias pulmonares.

Também foram medidos os fluxos na aorta ascendente. A performance e o volume de ambos os ventrículos fizeram parte desse estudo. Todos os fluxos foram comparados com os fluxos de crianças normais ${ }^{10}$.

\section{RESULTADOS}

Houve apenas 1 óbito no pós-operatório (paciente n: 5). Nesse caso, a relaçăo pressão sistólica do ventrículo direito (VD), pressão sistólica ventrículo esquerdo (VE), (VD/VE) medida no intra-operatório após a saída de circulação extracorpórea, foi de 0,52 ; as primeiras 24 horas do período de pós-operatório transcorreram sem complicações, com débito cardíaco adequado; entretanto, após esse período, o paciente apresentou parada cardiocirculatória irreversível. O estudo necroscópico revelou necrose do feixe de His e de seus ramos; o nó atrioventricular e a artéria coronária direita eram normais.

Nenhum paciente apresentou baixo débito cardíaco ou outras complicações pós-operatórias após a cirurgia.

Os 6 pacientes receberam alta hospitalar e permanecem assintomáticos após período de pós-operatório de 1 a 14 meses. Esses pacientes foram seguidos através de estudo ecocardiográfico seriado (Tabela 2). 0 ecocardiograma bidimensional mostrou diâmetro e volume normais em ambos os ventrículos. A válvula monocúspide se mostrou bem localizada e com funçăo normal (Figura 3). A insuficiência pulmonar, detectada ao ecodoppler, esteve presente nos 6 pacientes, sendo considerada moderada para importante em apenas 1 paciente; nos 5 pacientes restantes, a insuficiência pulmonar foi considerada leve. Nāo se detectou obstruçăo significativa na via de saída do ventrículo direito, através da válvula monocúspide ou na anastomose pulmonar. Cinco pacientes foram submetidos a cateterismo entre 15 e

TABELA 2

DADOS ECODOPPLERCARDIOGRÁFICOS POSS-OPERATÓRIOS

\begin{tabular}{|c|c|c|c|c|c|c|c|}
\hline Paciente & $\begin{array}{c}\text { Evoluçāo tardia } \\
\text { (meses) }\end{array}$ & $\begin{array}{l}\text { Insuficiéncia } \\
\text { pulmonar }\end{array}$ & $\begin{array}{c}\text { Velocidade de } \\
\text { fluxo em } T \text {. pulmonar } \\
\text { (cm/seg) }\end{array}$ & $\begin{array}{c}\text { Insuficiência } \\
\text { aórtica }\end{array}$ & $\begin{array}{l}\text { Velocidade de } \\
\text { fluxo em aorta } \\
\text { (cm/seg) }\end{array}$ & $\begin{array}{l}\text { Defeitos } \\
\text { residuais }\end{array}$ & $\begin{array}{l}\text { Fraçāo de } \\
\text { ejeçấo do VE }\end{array}$ \\
\hline 01 & 14 & Importante & 210 & Moderada & 160 & - & 0,87 \\
\hline 02 & 11 & Moderada & 125 & Pequena & 142 & $\begin{array}{c}\text { CIV } \\
\text { Pequena }\end{array}$ & 0,77 \\
\hline 03 & 07 & Pequena & 110 & Ausente & 130 & - & 0,82 \\
\hline 04 & 04 & Moderada & 130 & Pequena & 134 & - & 0,79 \\
\hline 06 & 02 & Pequena & 112 & Pequena & 128 & - & 0,83 \\
\hline 07 & 01 & Pequena & 100 & Pequena & 120 & - & 0,79 \\
\hline
\end{tabular}

VE: ventrículo esquerdo; CIV: comunicação interventricular. 


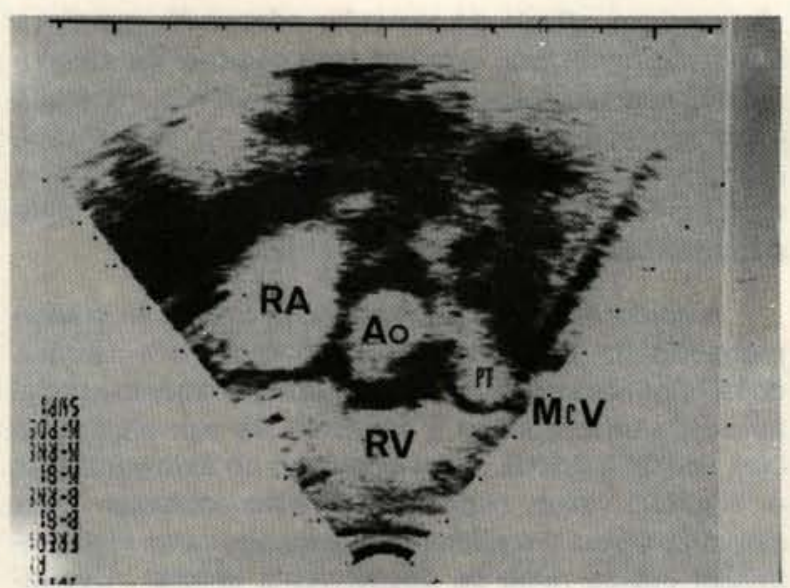

Fig. 3-Ecocardiograma pós-operatório: paciente de n: 3, corte no pequeno eixo ao nivel das valvas ventriculo-arteriais.

$\mathrm{RA}=$ átrio direito; $\mathrm{RV}=$ ventrículo direito; $\mathrm{Ao}=$ aorta; $\mathrm{McV}$ $=$ válvula monocúspide durante a diástole; $\mathrm{PT}=$ tronco pulmonar.

47 dias de pós-operatório (Tabela 3). A relação entre. as pressōes sistólicas ventrículo direito/ventrículo esquerdo foi moderadamente èlevada em apenas 1 paciente (caso $\mathrm{n}: 2$ ) associado, ainda, a uma pequena comunicação interventricular.

Não houve gradientes significativos entre o ventrículo direito e artérias pulmonares. Nenhum shunt intracardíaco foi detectado, exceto no paciente acima mencionado; não houve evidência angiográfica de distorção das artérias (Figura 4).

\section{DISCUSSÃO}

O uso de condutos extracardíacos permitiram o tratamento de várias anomalias cardíacas congênitas complexas $^{6}$. Porém, quando utilizados em neonatos e crian-

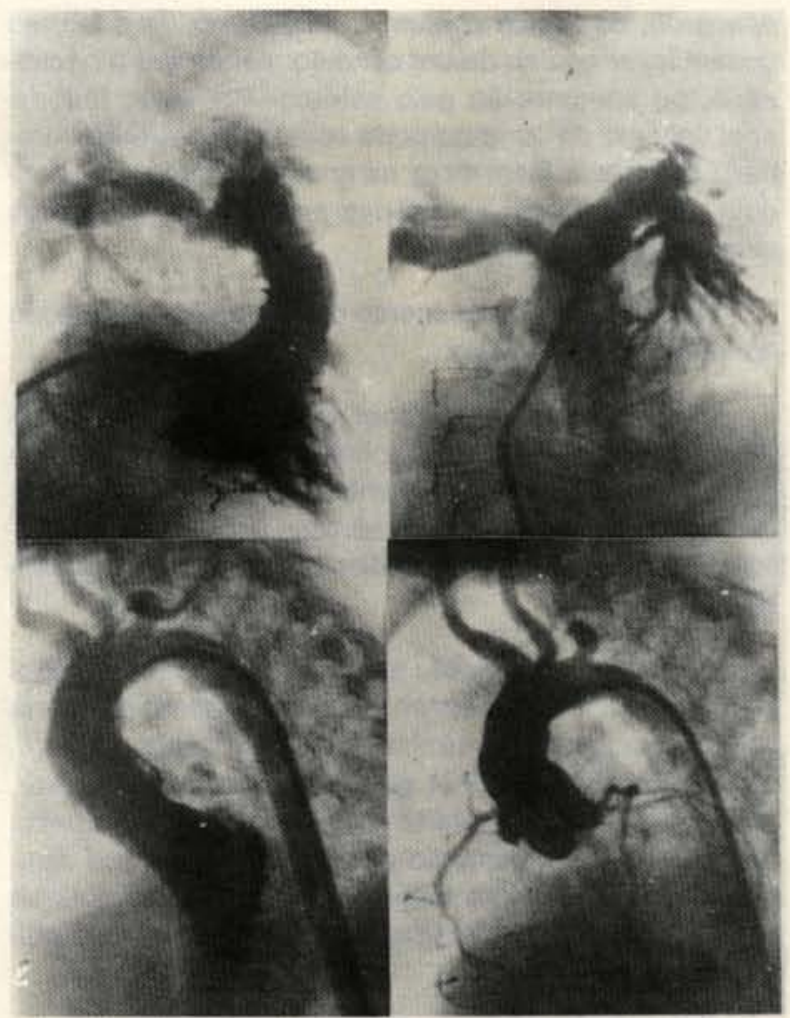

Fig. 4-Angiocardiografia pós-operatória. Nos quadros superiores, injeçâo no ventrículo direito e na via de saída; nos quadros inferiores, aspecto do ventrículo esquerdo e da aorta.

ças de baixo peso, são freqüentes e graves os problemas imediatos e tardios. No paciente portador de truncus arteriosus, vários fatores relacionados à doença dificultam a colocação do conduto. O baixo desenvolvimento ponderal, a cardiomegalia e a dilatação do tronco comum são fatores restritivos à colocação do conduto, levando-se em consideração, ainda, a presença do pulmão

TABELA 3

CATETERISMO POSS-OPERATORIO PRECOCE EM 5 PACIENTES

\begin{tabular}{|c|c|c|c|c|c|c|c|c|c|c|c|c|}
\hline \multirow{2}{*}{$\begin{array}{c}\text { Paciente } \\
\quad N^{0}\end{array}$} & \multicolumn{5}{|c|}{ Pressōes (mmHg) } & & \multirow{2}{*}{$\begin{array}{c}\text { Relaçáo da pressão } \\
\text { sistólica VDNE }\end{array}$} & \multicolumn{5}{|c|}{ Saturaçâo de $\mathrm{O}_{2}(\%)$} \\
\hline & $A D$ & $V D$ & $A P E$ & $A P D$ & $V E$ & Ao & & $A D$ & $V D$ & $A P D$ & $V E$ & Ao \\
\hline 01 & $v=3$ & $47 / 8$ & $38 / 0$ & $39 / 0$ & $102 / 3$ & $9 / 70$ & 0,49 & 66 & 64 & 65 & 90,5 & - \\
\hline & $\begin{array}{l}a=6 \\
\bar{m}=5\end{array}$ & 20 & $x_{12}$ & & & & & & & & & \\
\hline 02 & $\begin{array}{l}v=7 \\
a=9 \\
\tilde{m}=7\end{array}$ & $68 / 11$ & $60 / 3$ & $39 / 14$ & $113 / 10$ & - & 0,60 & 68 & 65 & 73 & 95 & 95 \\
\hline 03 & $\begin{array}{l}v=2 \\
a=4 \\
\bar{m}=3\end{array}$ & $35 / 5$ & $35 / 5$ & $31 / 3$ & $30 / 4$ & $74 / 6$ & 0,47 & 60 & 60 & 69 & 96 & 93 \\
\hline 04 & $\overline{\mathrm{m}}=5$ & $47 / 3$ & - & $33 / 0$ & $98 / 4$ & $80 / 60$ & 0,48 & 69 & 69 & 66 & 92 & - \\
\hline 06 & $\overline{\mathrm{m}}=8$ & $45 / 9$ & - & $50 / 19$ & $89 / 7$ & $83 / 60$ & 0,51 & 67 & 69 & 64,5 & 98 & 一 \\
\hline
\end{tabular}

AD: átrio direito; VD: ventrículo direito; APE: artéria pulmonar esquerda; APD: artéria pulmonar direita; VE: ventrículo esquerdo; Ao: aorta. 
esquerdo, do tronco comum e do esterno. Tais fatores podem levar ao uso de um conduto menor que o necessário, ou compressão pelo esterno. Por outro lado, o anel valvular do conduto pode comprimir a artéria coronária esquerda. Além disto, na anastomose distal, o conduto pode distorcer as artérias pulmonares, devido às suas delgadas paredes.

É inevitável o crescimento do pacientes em relação ao tamanho do conduto.

Calcificação valvular com estenose nos condutos de Dacron, estenoses na anastomose proximal ou no próprio tubo são freqüentemente observados tardiamente. Mesmo os homoenxertos aórticos podem sofrer calcificaçōes tardiamente, além dos problemas de disponibilidade.

A anastomose direta ventrículo-pulmonar permite a ocupaçāo de todo o espaço pelo tronco comum, artéria pulmonar esquerda e ventrículo direito. Desta maneira, a conexão VD - artéria pulmonar esquerda é maior, sem a expansão do retalho fora do contorno cardíaco. A chance de sangramento pós-operatório parece diminuir com o uso dessa técnica, pois as únicas suturas externas são aquelas correspondentes à circulação pulmonar.

A correção proposta não difere muito da técnica geralmente empregada para a correção da tétrade de Fallot com hipoplasia do anel e do tronco pulmonar, necessitando um retalho transanular. A probablidade de estenose do tronco é menor, pois a parede posterior da anastomose é feita com o tecido do próprio paciente, tendo a chance potencial de crescimento.

Algumas poucas tentativas de correção do tronco comum sem o uso de condutos extracardiacos foram referidas ${ }^{2}$, mas falharam em mostrar bons resultados. Apesar do uso de válvula monocúspide, a insuficiência pulmonar foi considerada responsável pelos resultados insatisfatórios. Por outro lado, com o uso de conduto extracardíaco não valvulado para correçăo do truncus em neonatos, PEETZ et alli ${ }^{7}$, concluíram que são compativeis com bons resultados tardios, apesar da insuficiência pulmonar residual. Na nossa opinião, devido à alta resistência arteriolar pulmonar nesses pacientes, a colocaçāo de uma monocúspide é particularmente necessária no período de pós-operatório imediato, até que haja uma progressiva normalização pressórica das artérias pulmonares. Por outro lado, pensamos que a coloca- ção de uma válvula de tamanho adequado, grande o suficiente para ocluir a maior área possível da anastomose ventrículo-pulmonar, é crítica. A válvula necessita estar colocada na sua porção mais alta no nivel arterial, de maneira que, durante o período de fechamento, a margem livre vai de encontro ao esporão correspondente à anastomose ventrículo-pulmonar (Figura 1e).

A causa mortis do paciente de n: 5 foi, com grande probabilidade, devido a um infarto septal e à necrose do feixe de His e seus ramos, já que o suprimento arterial ao tecido de condução é variável ${ }^{3}$. Da sua origem na crux cordis, a artéria nodal alcança o nó atrioventricular e, algumas vezes, supre o feixe atrioventricular. Uma segunda artéria é o primeiro ou segundo ramo interventricular septal, ramo da descendente anterior. Dependendo de variações anatômicas, essas artérias septais podem ser a única fonte arterial do feixe e seus ramos. Esta séria complicaçāo, conseqũente à ampliação da comunicação interventricular, chama nossa atenção quanto ao risco da ressecção septal, quando feita em pacientes com truncus.

A técnica proposta tem sua indicação ideal na maioria dos neonatos e crianças com truncus arteriosus tipo I ou II ( $86 \%$ dos casos) ${ }^{\prime}$, com insuficiência cardíaca congestiva e baixo peso. Neste grupo, a cerclagem das artérias pulmonares, ou a correção com condutos extracardiacos são seguidos de morbidade e mortalidade precoce e tardia.

Baseados nos resultados clínicos, hemodinâmicos e ecodopplercardiográficos, a técnica descrita parece a melhor escolha, nesse grupo de pacientes. Entretanto, uma evoluçāo maior é necessária, para se avaliarem, apropriadamente, os resultados tardios.

\section{ADENDA}

Após realização deste trabalho, foram operados outros 4 pacientes, com truncus tipo I. Três tiveram boa evolução, com resultados clínicos e ecocardiográficos semelhantes aos anteriores. Um paciente faleceu, subitamente, no $77^{\circ}$ dia de pós-operatório; apresentava comunicação interventricular restritiva, o que motivou sua ampliação com ressecção da porção anterior do septo interventricular; a necrópsia evidenciou extensa área de hemorragia septal. 
BARBERO-MARCIAL, M.; RISO, A. A.; VERGINELLI, G.; PILEGGI, F.; JATENE, A. D. - Technique for correction of truncus arteriosus type I and II without extracardiac conduits. Rev. Bras. Cir. Cardiovasc., 4(1): 1-8, 1989.

ABSTRACT: A new corrective surgery for truncus arteriosus (TA) without the use of extracardiac conduit was employed in 7 patients with truncus (TA) type I in 6 cases and type II in 1 case, aged from two to nine months. The common TA was septated with a patch into aortic and pulmonary segments and the ventricular septal defect was closed through ventriculotomy; a direct anastomosis between the pulmonary arteries and the right ventricle was performed, the anterior wall of this, being contructed with a patch with a monocusp valve. There was one imediate postoperative death. In the surviving six patients the RV/LV postoperative peak systolic was less than 0.51 in five and 0.60 in one with residual VSD; all are in functional class I, in postoperative period between one and fourteen months. Based in these results we propose this technique for patients with type I or II, in the first year of life.

DESCRIPTORS: truncus arteriosus, surgery.

\section{REFERÊNCIAS BIBLIOGRÁFICAS}

1 ARGAWAL, K. D.; EDWARDS, W. D.; FELDT, R. H.; DANIELSON, G. K.; PUGA, F. J.; McGOON, D. C. Clinicopathological correlated of obstructed rightsided porcine-valved extracardiac conduits. J. Thorac. Cardiovasc. Surg., 81(4): 591-601, 1981.

2 BAILEY, L. L.; PETRY, E. L.; DOROSHOW, E. W.; JACOBSON, J. C.; WAREHAN, E. - Biologic reconstruction of the right ventriculr outflow tract: preliminary experimental analysis and clinical application in a neonate with type I truncus arteriosus. J. Thorac. Cardiovasc. Surg., 82(5): 770-784, 1981.

3 DAVIES, M. J.; ANDERSON, R. H.; BECKER, A. R. Blood supply of the conduction tissues.. In: The conduction system of the heart. London, Butterworths, 1983. p. $74-80$.

4 KIRKLIN, J. W. \& BARRAT-BOYES, B. G. - Truncus arteriosus. In: Cardiac Surgery. New York, John Wiley \& Sons, 1986. Cap. 28, p. 911-931.

5 LECOMPTE, I.; NEVEUX, J. I.; LECA, F.; ZANINI, L.; DUBOIS, I. - Reconstruction of the pulmonary outflow tract without prosthetic conduit. J. Thorac. Cardiovasc. Surg., 84(5): 727-733,

6 McGOON, D. C.; WALLACE, R. B.; DANIELSON, G. K. - The Rastelli operation: its indications and results. J. Thorac. Cardiovasc. Surg., 65(1): 65-75, 1983.

7 PEETZ, D. J.; SPICER, R.; CROWLEY, D. C.; SLOAN, H.; BEHRENDT, D. M. - Correction of truncus arteriosus in the neonate using a nonvalved conduit. J. Tho rac. Cardiovasc. Surg., 83(5): 742-746, 1982.

8 SING, A K.; De LEVAL, M. R.; PINCOTT, J. R.; STARK, J. - Pulmonary artery banding for truncus arteriosus in the first year of life. Circulation, 54(Supl. 3): 17-19, 1976.

9 VAN PRAAGH, R. \& VAN PRAAGH, S. - The anatomy of common aorticopulmonary trunk (Truncus Arteriosus
Communis) and its embriologic implications: a study of 57 necropsy cases. Am. J. Cardiol., 16(3): 406-425, 1965.

10 WILSON, N.; GOLDBERG, S. J.; DICKSON, D. F. - Normal intracardiac and great artery blood velocity measurement by pulsed Doppler echocardiography. $B r$. Heart. J., 53(4): 451-458, 1985.

\section{Discussão}

\section{DR. IVO A. NESRALLA Porto Alegre, RS}

Desejo cumprimentar o Dr. Miguel Marcial e seus colaboradores, pelo magnífico trabalho apresentado. A técnica descrita é uma adaptação engenhosa e original da abordagem empregada na correção da tétrade de Fallot com hipoplasia do anel e do tronco pulmonar. Gostaria de discutir a problemática do emprego da monocúspide de pericárdio autógeno que, além de produzir graus variáveis de insuficiência pulmonar, sabidamente irá calcificar. Pergunto ao autor se não seria mais adequada a inserção de homoenxerto aórtico no trajeto VDAP, Poderia ser de tamanho compativel com o crescimento da criança, evitando qualquer grau de insuficiência pulmonar, que, sabidamente, é a grande causa de maus resultados a longo prazo. As atuais técnicas de preservação dos homoenxertos aórticos são bem mais promissoras a longo prazo, quando comparadas a outros materiais biológicos. Muito obrigado.

\section{DR. NILZO RIBEIRO Salvador, $B A$}

Serei bastante breve, com relação àquele paciente em que teve que ser interposta a aurícula esquerda e que teve morte súbita no pós-operatório; será que pode ter ocorrido a oclusão da descendente anterior? Esta é 

tipos I e II sem condutos extracardiacos. Rev. Bras. Cir. Cardiovasc., 4(1): 1-8, 1989.

uma pergunta que eu faria ao Dr. Miguel; uma preocupação que eu tenho é que, realmente, nessa área da artéria que sai do ventrículo direito ela é muito angustiada; será que a incisão na víscera não nos coloca muito próximos às porçōes iniciais da descendente anterior e, por isso, pode trazer alguns problemas com relação à artéria? Esta é uma dúvida que eu gostaria que o Dr Miguel nos tirasse. De qualquer maneira, estão aqui os meus parabéns, acho que todos nós estamos altamente sensibilizados com o nivel, com a seriedade do trabalho apresentado pelo Dr. Miguel e pela beleza, inclusive, da apresentação. Muito obrigado.

\section{DR. BARBERO-MARCIAL (Encerrando)}

Obrigado pelos carinhosos comentários do Dr. Nilzo e do Dr. Ivo, que, realmente, me sensibilizaram muito. Quanto à pergunta feita pelo Dr. Nilzo Ribeiro, por que faleceu aquele paciente, nós temos a necropsia; eu acho que a criança teve uma parada cardíaca súbita, 18 horas após a operação e a criança estava aceitavelmente bem, sem baixo débito, somente com dopamina. Nāo conse- guimos recuperá-la, levando para a sala e, com o auxílio da circulação extracorpórea, ficamos 2 horas tentando recuperá-la, mas foi impossivel e a necropsia mostrou infarto septal muito extenso. Essa criança tinha uma CIV restritiva e nós precisamos ressecar toda a parte inferior do septo interventricular, era muito grande a ressecção para poder direcionar o ventrículo esquerdo para a aorta; como disse, o tronco estava nascendo totalmente do ventrículo direito e, então, tivemos que ressecar todo o septo e tunelizar tudo para o anel da aorta e, nesse momento, acho, cortamos as artérias septais que irrigam o feixe de His e seus ramos direito e esquerdo, levando a uma necrose total do feixe de His e seus ramos, havendo o infarto septal. Eu acho que a causa da morte foi essa. Como se sabe, a irrigação do nó atrioventricular vem através de um ramo, em geral, da coronária direita, é uma irrigação diversa da do feixe de His. Quanto à pergunta do Dr. Ivo, nós também conhecemos bem os novos trabalhos de um enxerto aórtico, tem dado resultados melhores que os outros tubos. Mas a certeza da reoperação existe já na primeira operação, pois o enxerto não cresce e, então, nós preferimos optar por uma operação que não precise tubos, porque, com esta nova operação, temos bastante chance de que não exista necessidade de reoperação futura. Muito obrigado. 